\title{
Theory of Mirror Spectographs III. Focal Surfaces and Slit Curvature of Ebert and Ebert-
Fastie Spectographs
}

\author{
Klaus D. Mielenz
}

(July 28, 1964)

\begin{abstract}
A spatial theory of the focal surfaces and slit curves of Ebert and Ebert-Fastie monochromators and spectrographs is presented.

In a second-order approximation, it yields closed expressions for the focal surface, from which a "stop-shift" theory is developed to study the influence of the position of the grating in the spectrograph upon the curvature of the spectrum. The condition for an extended flat spectrum is derived, and the Ebert-Fastie system is shown to be the preferable one for spectrograph design.

The curvature of long monochromator slits is also derived as a closed, second-order expression. The Ebert system, only, can be equipped with long slits, so that it, in turn, constitutes the superior monochromator mounting.

A fourth-order approximation is also included for still more accurate computations.
\end{abstract}

\section{Introduction}

In two previous papers $[1,2],{ }^{1}$ a general theory was developed for calculating the focal surfaces and slit curv ss of Czerny-Turner type mirror spectrographs and monochromators. This theory will now be applied to Ebert and Ebert-Fastie systems with either plane reflectance gratings or Littrow prisms as dispersive elements (though gratings, only, will be explicitly treated in the following).

\section{Exact Theory}

\subsection{Basic Expressions}

In Ebert and Ebert-Fastie monochromators and spectrographs ([3, 4]; fig's. 1 and 2 ), a single concave mirror is used to fulfill the tasks of both collimator and camera. Let $r$ be the radius of curvature of the mirror, which we assume to be spherical.

A coordinate system (fig. 3), with its origin at the center of curvature, $M$, of the mirror, is chosen so that the $x$-axis intersects the grating center $O$, the $y$-axis encloses an angle $\theta$ with the grating tangent vector

$$
\mathbf{T}=(\sin \theta, \cos \theta, 0),
$$

and the $z$-axis is parallel to the grating grooves.

Then, let

$$
\mathbf{m}=m \mathbf{i},
$$

\footnotetext{
1 Figures in brackets indicate the literature references at the end of this paper.
}

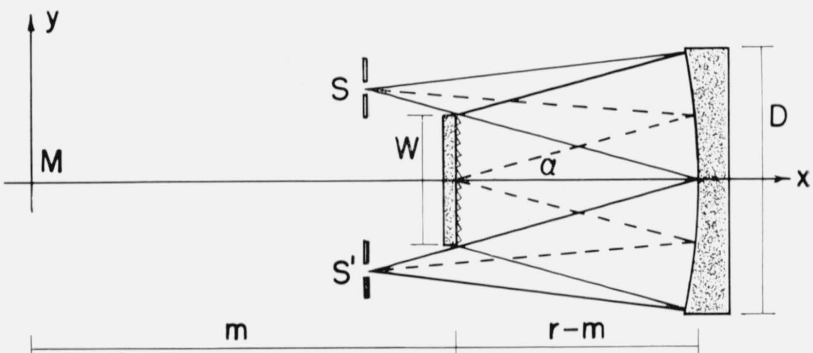

(a)

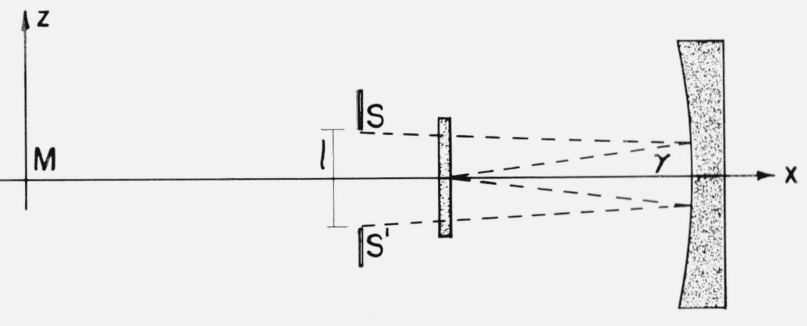

(b)

FiguRE 1. Ebert monochromator.

(a) Horizontal cross section with grating in zern-order position. (b) Vertical cross section; principal ray from upper end of entrance slit, $S$, to lower end of exit slit, $S^{\prime}$, is shown. 


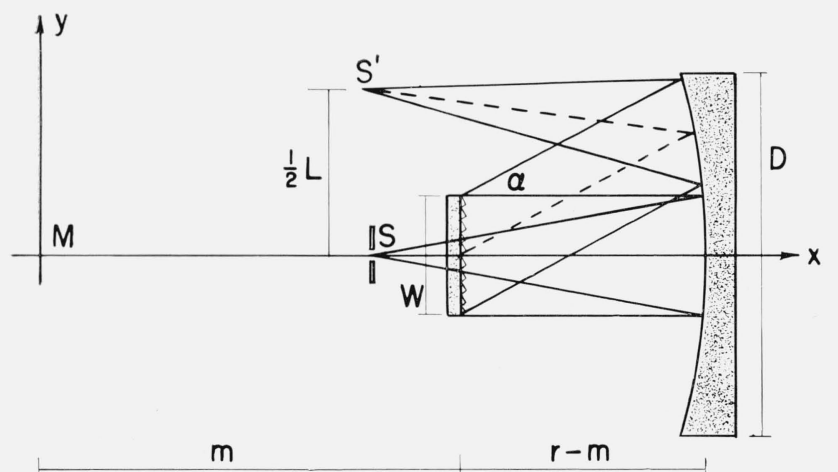

(a)

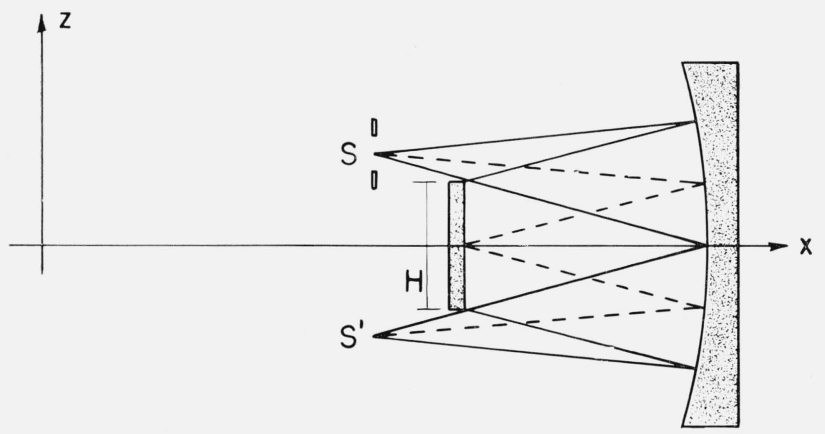

(b)

FiguRE 2. Ebert-Fastie spectrograph.

(a) Horizontal cross section with grating in symmetrical position; $s^{\prime}$ is outermost line of spectrum. (b) Vertical cross section.

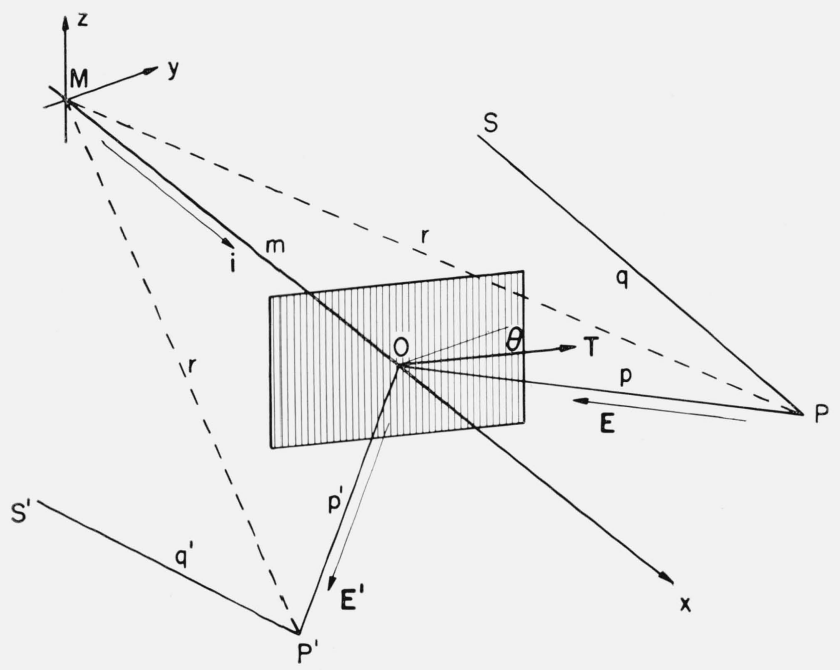

FiguRE 3. One-mirror spectrograph.

Beam path and coordinate system. with $\mathbf{i}=(1,0,0)$, be the point vector $\overrightarrow{O M}$, and let

$$
\begin{aligned}
\mathbf{E} & =(A, B, C), \\
\mathbf{E}^{\prime} & =\left(A^{\prime}, B^{\prime}, C^{\prime}\right),
\end{aligned}
$$

represent the unity vectors of the principal rays incident upon and diffracted by the grating at $O$.

From eqs $(2.7,2.5,2.17$, and 2.4$),{ }^{2}$ the point vector, $\mathbf{s}$, of a slit point $S$ is then obtained as

$\mathbf{s}=\overrightarrow{M S}=(x, y, z)=-\left(p+q-2 p q w / r^{2}\right) \mathbf{E}$

with

$$
+\left(1-2 q w / r^{2}\right) m \mathbf{i},
$$

$$
\begin{aligned}
& p=w+m A, \\
& q=\frac{1}{2} w+m^{2} C^{2} /(2 w), \\
& w=+\sqrt{r^{2}-m^{2}\left(1-A^{2}\right) .}
\end{aligned}
$$

With (2.6), the direction of the principal ray leaving $S$ is givein by the unity vector

$$
-\mathbf{q} / q=-\overrightarrow{P S} /|P S|=\left(1-2 p w / r^{2}\right) \mathbf{E}+\left(2 m w / r^{2}\right) \mathbf{i},
$$

where $P$ is the point of reflection on the collimator portion of the mirror.

Likewise, one obtains from [2], for the image point $S^{\prime}$,

$$
\begin{aligned}
& \mathbf{s}^{\prime}=\overrightarrow{M S^{\prime}}=\left(x^{\prime}, y^{\prime}, z^{\prime}\right)=\left(p^{\prime}+q^{\prime}-2 p^{\prime} q^{\prime} w^{\prime} / r^{2}\right) \mathbf{E}^{\prime} \\
&+\left(1-2 q^{\prime} w^{\prime} / r^{2}\right) m \mathbf{i}, \\
& p^{\prime}= w^{\prime}-m A^{\prime}, \\
& q^{\prime}=\frac{1}{2} w^{\prime}+m^{2} C^{\prime 2} /\left(2 w^{\prime}\right), \\
& w^{\prime}=+\sqrt{r^{2}-m^{2}\left(1-A^{\prime 2}\right),} \\
& \mathbf{q}^{\prime} / q^{\prime}=\overrightarrow{P^{\prime} S^{\prime} /\left|P^{\prime} S^{\prime}\right|}=\left(1-2 p^{\prime} w^{\prime} / r^{2}\right) \mathbf{E}^{\prime} \\
&-\left(2 m w^{\prime} / r^{2}\right) \mathbf{i} .
\end{aligned}
$$

We see that the equations for $S^{\prime}$ are the same as those for $S$, with $-\mathbf{E}^{\prime}$ substituted for $\mathbf{E}$. Hence, $S$ and $S^{\prime}$ lie on a common focal surface, and it suffices to consider $S$, only, to determine this surface.

Separate expressions for $S$ and $S^{\prime}$ are then merely needed to find the location of conjugate points on the focal surface. $S^{\prime}$ is a spectral image of $S$ when the grating equations,

$$
\begin{gathered}
\mathbf{T} \cdot\left(\mathbf{E}-\mathbf{E}^{\prime}\right)+\mu \lambda / d=0, \\
C-C^{\prime}=0,
\end{gathered}
$$

are satisfied ( $\mu=$ spectral order, $\lambda=$ wavelength, $d=$ grating constant; see reference [1]).

${ }^{2}$ Equations (7), (5), etc., of reference [2]. 


\subsection{Focal Surface}

From (4) through (8), one derives

$$
\mathbf{s}=-a \mathbf{E}+b \mathbf{i},
$$

with

$$
\begin{gathered}
a=\left(w^{2}+m^{2} C^{2}\right) /(2 w)+m^{2} B^{2}(w+m A) / r^{2}, \\
b=m^{3} B^{2} / r^{2},
\end{gathered}
$$

as a vector representation of the focal surface. Hence, for the coordinates of $S$,

$$
x=-a A+b, y=-a B, z=-a C .
$$

From here, a closed expression is obtained in two limiting cases, only:

For $m=0$ (grating at center of curvature of mirror), the above equations yield

$$
\mathbf{s}^{2}=x^{2}+y^{2}+z^{2}=\left(\frac{1}{2} r\right)^{2},
$$

which describes a sphere of radius $\frac{1}{2} r$ about $M$.

For $m=r$ (grating at vertex of mirror), one obtains

$$
\left(x-\frac{3}{4} r\right)^{2}+y^{2}+z^{2}\left(x-\frac{1}{2} r\right) /(x-r)=\left(\frac{1}{4} r\right)^{2} .
$$

At the paraxial focus, where $z^{2}\left(x-\frac{1}{2} r\right)$ is negligibly small, this describes a cylinder of radius $\frac{1}{4} r$ about the axis $x=\frac{3}{4} r, y=0$.

Yet, no workable closed expression for the focal surface can be derived for arbitrary values of $m$. The following parameter representation may be used instead.

Write, as a general expression for $\mathbf{E}$,

$$
\begin{aligned}
& A=-\cos \alpha, \\
& B=\mp \sqrt{\sin ^{2} \alpha-\sin ^{2} \gamma}, \\
& C=-\sin \gamma,
\end{aligned}
$$

where the choice of signs is due to the negative direction of $\mathbf{E}$ in figure 3. Hence, from (13),

$$
\begin{aligned}
& x=a \cos \alpha+b, \\
& y= \pm a \sqrt{\sin ^{2} \alpha-\sin ^{2} \gamma}, \\
& z=a \sin \gamma,
\end{aligned}
$$

where

$$
\begin{aligned}
& a=\left(r^{2} / 2 w\right) \\
& -\left[(1 / 2 w)-(w-m \cos \alpha) / r^{2}\right] m^{2}\left(\sin ^{2} \alpha-\sin ^{2} \gamma\right), \\
& b=\left(m^{3} / r^{2}\right)\left(\sin ^{2} \alpha-\sin ^{2} \gamma\right),
\end{aligned}
$$

and

$$
w=+\sqrt{r^{2}-m^{2} \sin ^{2} \alpha}
$$

are functions of $m, \alpha$, and $\gamma$.

\subsection{Slit Curve}

The curvature of long monochromator slits, as derived in [2] from the grating equations, is now obtained as follows:

Let the above eqs $(17 \mathrm{a}, \mathrm{b}, \ldots$. .) represent an arbitrary point, $S=(x, y, z)$, of the entrance slit. Then, let the slit center, $S_{0}=\left(x_{0}, y_{0}, z_{0}\right)$, be given by

$$
\begin{aligned}
& x_{0}=a_{0} \cos \alpha_{0}+b_{0}, \\
& y_{0}= \pm a_{0} \sqrt{\sin ^{2} \alpha_{0}-\sin ^{2} \gamma_{0},} \\
& z_{0}=a_{0} \sin \gamma_{0},
\end{aligned}
$$

with

$a_{0}=\left(r^{2} / 2 w_{0}\right)$

$-\left[\left(1 / 2 w_{0}\right)-\left(w_{0}-m \cos \alpha_{0}\right) / r^{2}\right] m^{2}\left(\sin ^{2} \alpha_{0}-\sin ^{2} \gamma_{0}\right)$,

$b_{0}=\left(m^{3} / r^{2}\right)\left(\sin ^{2} \alpha_{0}-\sin ^{2} \gamma_{0}\right)$,

$w_{0}=+\sqrt{r^{2}-m^{2} \sin ^{2} \alpha_{0}}$.

It was shown in [2], eq (2.21), that, as a consequence of the grating equations, the first direction cosine of $\mathbf{E}$ is the same for all slit points. Thus we may write in eqs $(17 \mathrm{a}, \mathrm{b}, \ldots .$.$) ,$

so that

$$
\alpha_{0}=\alpha_{0}, w=w_{0},
$$

$a=a_{0}+m^{2}\left[\left(1 / 2 w_{0}\right)-\left(w_{0}-m \cos \alpha_{0}\right) / r^{2}\right]\left(\sin ^{2} \gamma-\sin ^{2} \gamma_{0}\right)$,

$b=b_{0}-\left(m^{3} / r^{2}\right)\left(\sin ^{2} \gamma-\sin ^{2} \gamma_{0}\right)$.

Hence,

$$
\begin{aligned}
& x=x_{0}+\left(a-a_{0}\right) \cos \alpha_{0}+\left(b-b_{0}\right), \\
& y=y_{0} \pm a \sqrt{\sin ^{2} \alpha_{0}-\sin ^{2} \gamma} \mp a_{0} \sqrt{\sin ^{2} \alpha_{0}-\sin ^{2} \gamma_{0}}, \\
& z=z_{0}+a \sin \gamma-a_{0} \sin \gamma_{0},
\end{aligned}
$$

which, upon introduction of an additional parameter $\delta$,

$$
\gamma=\gamma_{0}+\delta
$$

is the parametric representation of a curve on the focal surface along which the entrance slit must extend.

It is then seen from eqs $(2.25$ and 26$)$ that, on the image portion of the focal surface, the exit slit must lie on the same curve.

\subsection{Discussion}

It was shown in 2.2 that different values of $m$ may yield entirely different focal surfaces. Proper positioning of the grating is therefore an important consideration in designing the spectrograph.

For a given $m$, the above parameter representations permit a straightforward point-by-point calculation 
of the focal surface and the slit curve. Hence, an electronic computer may conceivably perform an analysis of the influence of $m$ upon the system.

Closed expressions for arbitrary values of $m$, however, would provide a still better insight. Such expressions will be derived in the following chapter as a second-order approximation for the paraxial portion of the focal surface, which alone is of interest.

\section{Second-Order Approximation}

\subsection{Focal Surface - Choice of Grating Position}

Upon neglecting third-order terms in $\alpha$ and $\gamma$, eqs (16) and (12) may be written as

$$
\begin{aligned}
& A=-1 \pm \frac{1}{2} \alpha^{2}, \\
& B=\mp \sqrt{\alpha^{2}-\gamma^{2}}, \\
& C=-\gamma,
\end{aligned}
$$

$a / r=\frac{1}{2}+\frac{1}{4}\left[3(m / r)^{2}-4(m / r)^{3}\right] \alpha^{2}-\frac{1}{2}\left[(m / r)^{2}-2(m / r)^{3}\right] \gamma^{2}$,

$b / r=(m / r)^{3}\left(\alpha^{2}-\gamma^{2}\right)$.

Hence, as a second-order approximation of (13) for the paraxial region,

$$
\begin{aligned}
& x / r=\frac{1}{2}-\frac{1}{4}\left[1-3(m / r)^{2}\right] \alpha^{2}-\frac{1}{2}(m / r)^{2} \gamma^{2}, \\
& y / r= \pm \frac{1}{2} \sqrt{\alpha^{2}-\gamma^{2}}, \\
& z / r=\frac{1}{2} \gamma
\end{aligned}
$$

which is easily seen to satisfy the relation

$$
\begin{aligned}
f(x, y, z)=(x / r)+[1 & \left.-3(m / r)^{2}\right](y / r)^{2} \\
+ & {\left[1-(m / r)^{2}\right](z / r)^{2}-\frac{1}{2}=0 . }
\end{aligned}
$$

The usual discussion [5] shows that this equation represents

an elliptical paraboloid for $0<m<r / \sqrt{3}$,

a parabolic cylinder for $m=r / \sqrt{3}$,

a hyperbolic paraboloid for $r / \sqrt{3}<m<r$.

At a point $S=(x, y, z)$, the vector

$$
\mathbf{n}=-(\partial f / \partial x, \partial f / \partial y, \partial f / \partial z)
$$

or, from (23),

$$
\mathbf{n}=-\left(1 / r, 2\left[1-3(m / r)^{2}\right] y / r^{2}, 2\left[1-(m / r)^{2}\right] z / r^{2}\right)
$$

is normal to the focal surface [6]. The equation of the tangential plane of the focal surface at $S$, then, is

$$
(\xi-x, \eta-y, \zeta-z) \cdot \mathbf{n}=0
$$

or, with (25)

$$
\begin{aligned}
& (\xi-x)+2\left[1-3(m / r)^{2}\right](y / r)(\eta-y) \\
& +2\left[1-(m / r)^{2}\right](z / r)(\zeta-z)=0,
\end{aligned}
$$

where $\xi, \eta$, and $\zeta$ are the coordinates of a point in the plane [6]. These equations will be needed in the following discussion.

The horizontal cross sections of the paraxial focal surface (23), along which the spectrum extends, are

$$
(x / r)+\left[1-3(m / r)^{2}\right](y / r)^{2}=\frac{1}{2}, \quad z=0 .
$$

As shown in figure $4 \mathrm{a}$ for different values of $m$, they are parabolas whose curvature vanishes for

$$
m=r / \sqrt{3}
$$

and assumes opposite signs for greater and smaller values of $m$.

H. Ebert [3] had originally placed the grating at the paraxial focus of the mirror, where $m=\frac{1}{2} r$. O. Vierle [7] claims that perpendicular light incidence upon the focal surface is the advantage of this mounting. It is true that the direction of the principal ray, which from (8) can be shown to be

$-\mathbf{q} / q=-\left(1-\frac{1}{2}\left[1+2(m / r)^{2}\right] \alpha^{2}\right.$,

$$
\left.[1-2(m / r)] \sqrt{\alpha^{2}-\gamma^{2}},[1-2(m / r)] \gamma\right)
$$

is perpendicular to the $y z$-plane for $m=\frac{1}{2} r$. The focal surface, however, is curved away from that plane, and the angle of incidence, $\tau$, is therefore given by

$$
\cos \tau=(\mathbf{n} \cdot \mathbf{q}) /(n q),
$$

with $n=|\mathbf{n}|$. From (25) and (29),

$\cos \tau=1-\frac{1}{2}(m / r)^{2}\left[4-12(m / r)+9(m / r)^{2}\right] \alpha^{2}$

$$
-4(m / r)^{3}[1-(m / r)] \gamma^{2} .
$$

Except for the border case $m=0$, normal incidence occurs for no value of $m$, and $m=\frac{1}{2} r$ does not appear to be distinguished from other values. The numerical example in figure 5 shows, moreover, that $\tau$ is a very small angle, so that the whole matter is of little practical importance.

R. F. Jarrell [4] chose $m=\frac{3}{4} r$, with the grating midway between paraxial focus and mirror, so that the mirror size could be reduced. According to figure $1 \mathrm{a}$, the mirror diameter must be at least

$$
D=W+2(r-m) \alpha,
$$

in the horizontal cross section of an Ebert monochromator, where $W$ is the grating width and $\alpha$ the maximum angle. The same formula holds for the Ebert-Fastie spectrograph also; see figure 2 a. Hence, an increase of $m$ will indeed considerably reduce the mirror size. 


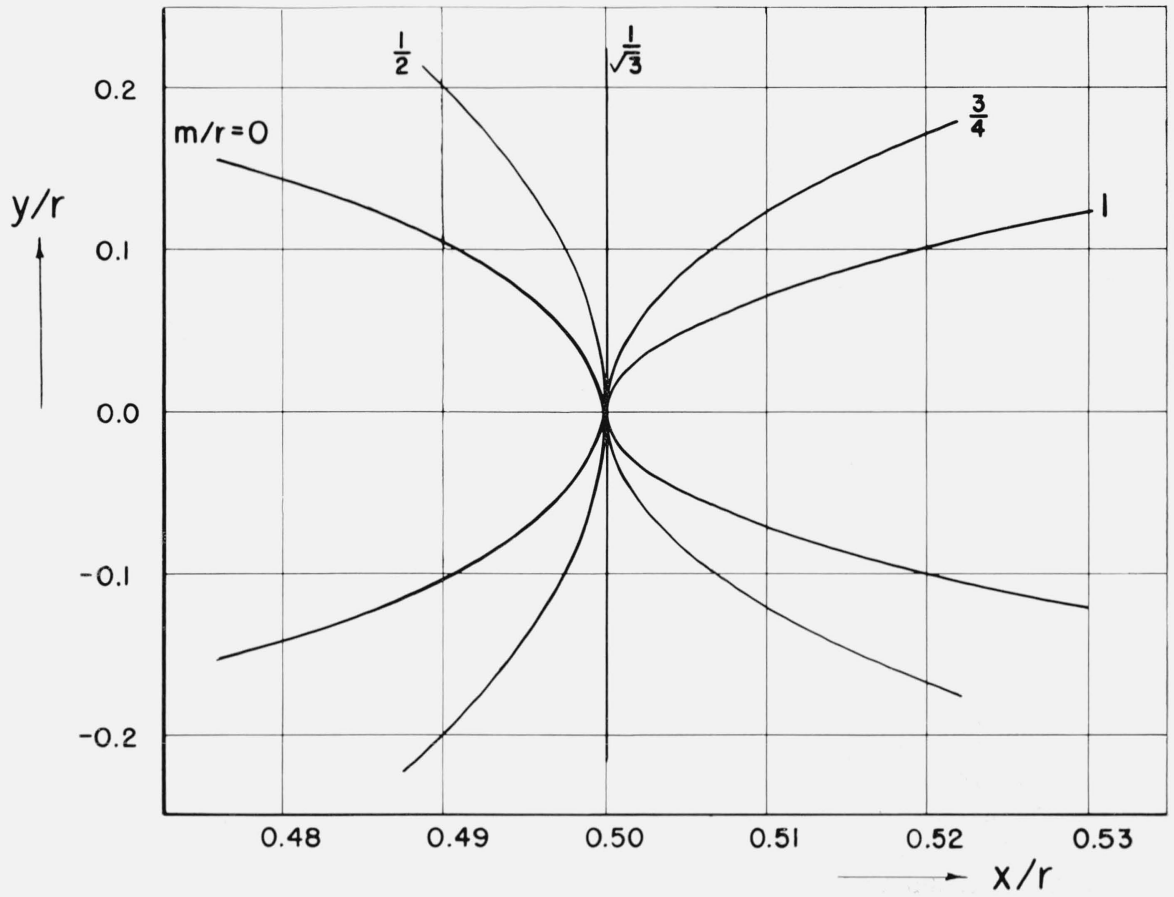

(a)

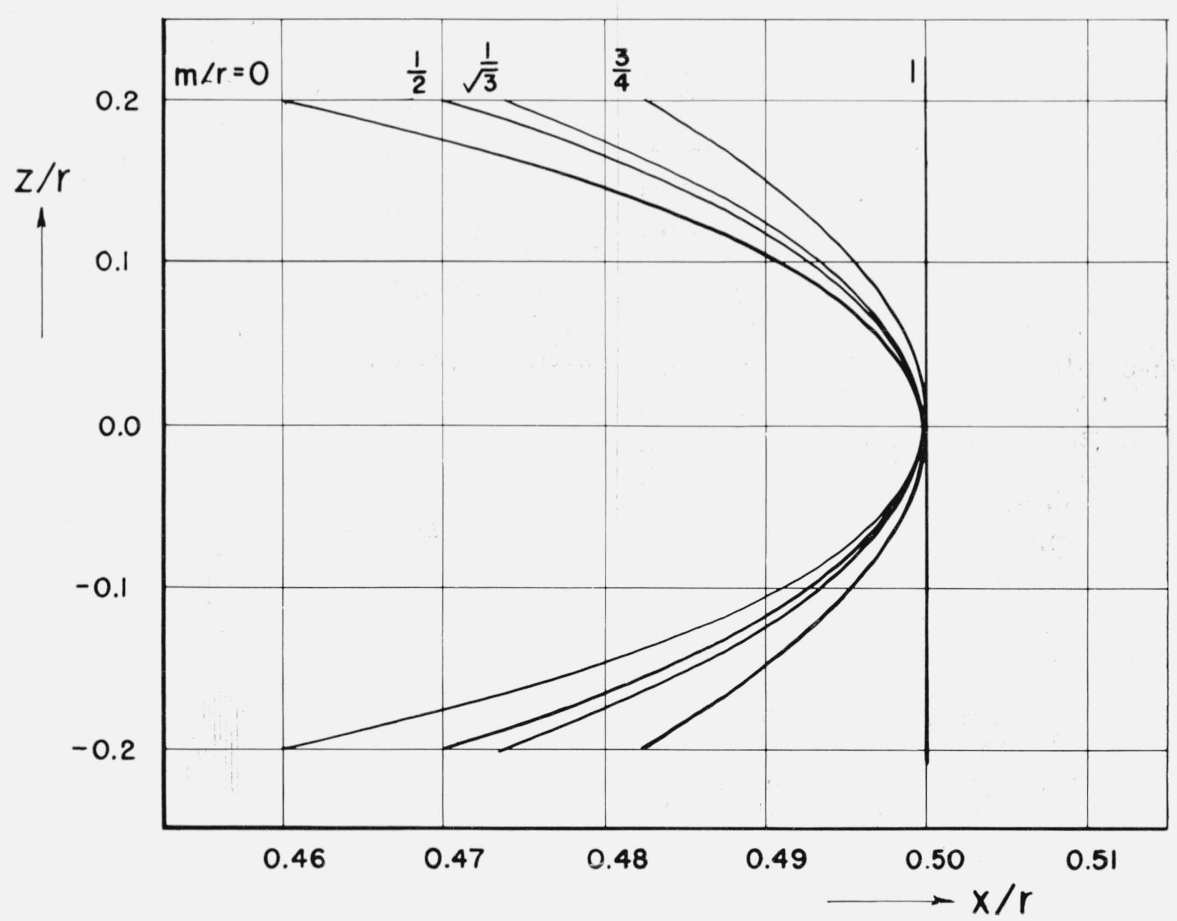

(b)

Figure 4. (a) Horizontal and (b) vertical cross sections of paraxial focal surface for different grating positions $\mathrm{m}$.

Scale of abscissa is 10 times enlarged. 


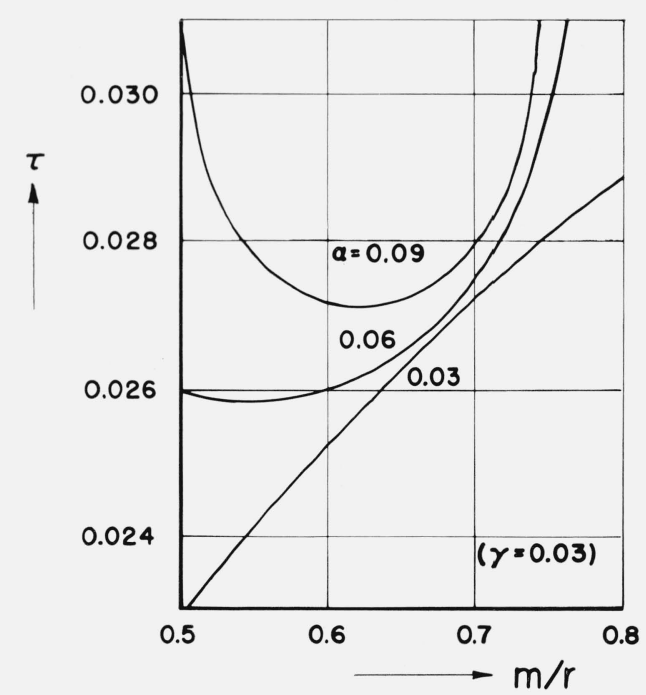

Figure 5. Angle of incidence on focal surface, $\tau$, versus grating position, $\mathrm{m}$.

Yet, a curved focal surface is obtained unless the " $\sqrt{3}$-condition" (28) is observed. This condition, which was first published by S. A. Khrshanovskii [8], holds as a second-order approximation and is now seen to apply to horizontal cross sections of the focal surface, only.

The vertical cross sections,

$$
(x / r)+\left[1-(m / r)^{2}\right](z / r)^{2}=\frac{1}{2}, \quad y=0,
$$

as shown in figure $4 \mathrm{~b}$, all curve away from the mirror. Their curvature decreases as the grating moves toward the mirror, and vanishes in the limiting case $m=r$, only.

A truly flat spectrum, in the sense of the focal surface being a plane, can therefore not be produced at all. Yet, the $\sqrt{3}$-condition defines the only position of the grating for which a plane photographic plate can be made tangent to the focal surface along a whole straight line, instead of at a single point, only, as for all other choices of $m$. It is seen from (26) that the photographic plate must then be arranged in the plane

$$
\left(\xi-x_{0}\right) / r+\frac{4}{3}\left(z_{0} / r\right)\left(\zeta-z_{0}\right) / r=0,
$$

where $x_{0}, y_{0}=0$, and $z_{0}$ are the coordinates of the center point of the central line.

In a spectrograph, the spectrum lines are usually short, so that the above described removal of the horizontal curvature of field, only, is fully sufficient to yield a flat spectrum.

\subsection{Slit Curve-In-Plane and Off-Plane Monochromators}

It is seen from eqs (22) that, upon retention of second-order terms in $\alpha$ and $\gamma$, only,

$$
\left(x-\frac{1}{2} r\right)^{2}+y^{2}+z^{2}=\frac{1}{4} r^{2} \sin ^{2} \alpha .
$$

If, in this equation, $x, y$, and $z$ are assumed to represent an arbitrary point of the entrance slit of a monochromator, the right-hand side of the equation is seen, from (19a), to be a constant;

$$
\frac{1}{4} r^{2} \sin ^{2} \alpha=\frac{1}{4} r^{2} \sin ^{2} \alpha_{0}=\rho_{0}^{2},
$$

where $\alpha_{0}$ pertains to the slit center. Thus,

$$
\left(x-\frac{1}{2} r\right)^{2}+y^{2}+z^{2}=\rho_{0}^{2} .
$$

This is the equation of a sphere of radius $\rho_{0}$ about the paraxial focus $\left(\frac{1}{2} r, 0,0\right)$. As set forth in [2], the exit slit, too, must obey this equation.

The slits must of course lie on the focal surface of the monochromator as well. They must therefore extend along the curve which is obtained as the section of the focal surface (23) with the sphere (34b).

In the vertical cross section

$$
x=\frac{1}{2} r,
$$

the slit curve is a circle about the paraxial focus $(y=z=0)$;

$$
y^{2}+z^{2}=\rho_{0}^{2},
$$

see figure 6. W. G. Fastie has, indeed, greatly improved the image quality of an Ebert monochromator by curving the two slits along this circle $[9,10]$. A further improvement might be accomplished by fitting the slits to the true spatial slit curve, as derived above.

In an Ebert-Fastie system, the slit curve intersects the slit center, $S_{\mathrm{EF}}$ in figure 6 , in a direction perpendicular to the grating grooves; i.e., perpendicular to the natural direction of the spectrum lines. It must be concluded, therefore, that the Ebert-Fastie mounting cannot possibly be equipped with long slits.

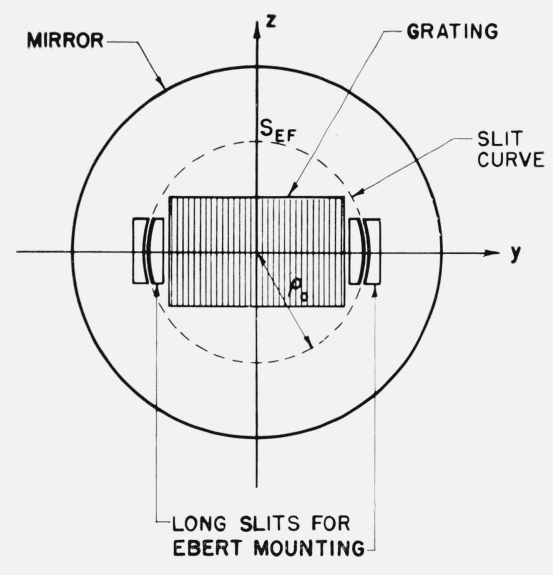

Figure 6. End-on view of one-mirror monochromator and slit curve. 


\subsection{Validity of Approximation}

The angles $\alpha$ and $\gamma$ that occur in practical spectrograph design may be determined as follows.

The angle $\alpha$ at which entrance and exit slits are arranged in a properly designed Ebert monochromator (where $\alpha$ is minimized) is obtained from figure 1a as

$$
\alpha \sim \tan \alpha=\frac{1}{2} W /(r-m),
$$

where $W$ is the grating width. The other angle, $\gamma$, is determined by the slit length, $l$. From figure $1 \mathrm{~b}$ and eq $(22 \mathrm{c})$,

$$
z=\frac{1}{2} l \sim \frac{1}{2} r_{\gamma}, \gamma \sim l / r,
$$

for the slit ends.

A high-speed Ebert monochromator with a long slit $\left(W=16^{\prime \prime}, l=4.5^{\prime \prime}, r=180^{\prime \prime}, m=\frac{1}{2} r\right)$ was constructed by W. G. Fastie [10], and a further enhancement of speed or slit length appears to be unlikely in the present state of the art. The corresponding angles are $\alpha=0.09, \gamma=0.025$.

In an Ebert-Fastie spectrograph, $\gamma$ is given in the same manner as $\alpha$ is in an Ebert monochromator, but with the grating height, $H$, substituted for $W$; see figure $2 \mathrm{~b}$. Thus,

$$
\gamma \sim \frac{1}{2} H /(r-m) .
$$

For $\alpha$, the spectrum length, $L$, is the determining factor. From figure 2a and eq (22b), for the spectrum ends,

$$
y=\frac{1}{2} L \sim \frac{1}{2} r \sqrt{\alpha^{2}-\gamma^{2}}, \alpha \sim \sqrt{(L / r)^{2}+\gamma^{2}},
$$

with $\gamma$ from (37a).

For two published designs of Ebert-Fastie spectrographs (R. F. Jarrell [4]: $r=22^{\prime}, H=2 \frac{1}{2}^{\prime \prime}, \quad m=\frac{3}{4} r$; P. Kroeplin [11]: $r=4 \mathrm{~m}, H=5 \mathrm{~cm}, m$ (estimated) $=$ $\frac{1}{2} \mathrm{r}$ ), we obtain $\gamma=0.02$ and 0.01 , respectively. Jarrell chose $L=20^{\prime \prime}$, or $L / r=0.076$, and image quality at the spectrum ends seems to indicate that this constitutes an upper limit [12]. Kroeplin uses $L=30 \mathrm{~cm}$ and, thus, $L / r=0.075$. From here and with the above values of $\gamma, \alpha=0.076$ and 0.078 , respectively.

We may thus accept

$$
\alpha=0.1, \gamma=0.03
$$

as maximum values for both Ebert monochromators and Ebert-Fastie spectrographs. A computation of the focal surface, from both the exact formulas (17) and the second-order approximation (22), then shows that the latter involves a maximum error of approximately

$$
\Delta S=\sqrt{(\Delta x)^{2}+(\Delta y)^{2}+(\Delta z)^{2}}=5 \cdot 10^{-5} r
$$

at these maximum angles and for various values of $m$ between $\frac{1}{2} r$ and $\frac{3}{4} r$. Here $\Delta S$ is the distance of the approximate focal point from the true one.

For the usual mirror radii of a few meters this error is of the order of $0.1 \mathrm{~mm}$, and it may therefore be concluded that the second-order approximation is sufficiently exact for most practical applications of the theory.

\section{Fourth-Order Approximation, Flat Spectra}

Where still more accuracy is needed, a fourthorder approximation of the focal surface may be used. If, in (21a, b, and c), the next higher terms in $\alpha$ and $\gamma$ are also retained one finds, instead of $(22 \mathrm{a}, \mathrm{b}$, and $\mathrm{c})$,

$$
\begin{aligned}
& x / r= \frac{1}{2}\left[1-(m / r)^{2} \gamma^{2}+\frac{1}{3}(m / r)^{2} \gamma^{4}\right] \\
&- \frac{1}{4}\left\{\left[1-3(m / r)^{2}\right]-\left[(m / r)^{2}-4(m / r)^{3}+3(m / r)^{4}\right] \gamma^{2}\right\} \alpha^{2} \\
&+\frac{1}{48}\left[1-30(m / r)^{2}+48(m / r)^{3}-27(m / r)^{4}\right] \alpha^{4}, \quad(39 \mathrm{a}) \\
& y / r= \pm \frac{1}{2} \sqrt{\alpha^{2}-\gamma^{2}}\left\{1-\frac{1}{6}\left[1+6(m / r)^{2}-12(m / r)^{3}\right] \gamma^{2}\right. \\
&\left.\quad-\frac{1}{6}\left[1-9(m / r)^{2}+12(m / r)^{3}\right] \alpha^{2}\right\}, \quad(39 b) \\
& z / r=\frac{1}{2} \gamma\left\{1-\frac{1}{6}\left[1+6(m / r)^{2}-12(m / r)^{3}\right] \gamma^{2}\right. \\
&\left.\quad+\frac{1}{2}\left[3(m / r)^{2}-4(m / r)^{3}\right] \alpha^{2}\right\} . \quad(39 \mathrm{c})
\end{aligned}
$$

These equations permit a point-by-point computation of the focal surface and, upon use of (19a), of the slit curve as well. The deviation from the true focal surface is

$$
\Delta S \sim 2 \cdot 10^{-7} r,
$$

for $\alpha=0.1, \gamma=0.03$, and $\frac{1}{2} r<m<\frac{3}{4} r$, as in 3.3. This is equivalent to about $1 \mu$, only, for a mirror radius of a few meters, so that the fourth-order approximation will certainly suffice for any application of the theory.

Equation (39a) shows that, for $m=r / \sqrt{3}$, the $x$-coordinates of spectrum center and ends $(\alpha=\gamma$ and $\pm \alpha_{\max }$, respectively) differ by fourth-order terms. With the grating in the $\sqrt{3}$-position, the horizontal cross sections of the focal surface are therefore still slightly curved.

The flatness of the spectrum may now be further improved by slightly correcting the grating position by an amount $\epsilon$ for which, in the fourth-order approximation of (39a), the spectrum center and the spectrum ends lie on a straight line. Thus, introduce

$$
m=r / \sqrt{3}+\epsilon
$$

into (39a), calculate $x=x(\epsilon)$ for $\alpha=\gamma$ and $\alpha= \pm \alpha_{\max }$, and equate the two $x$-values so obtained in order to find $\epsilon$. 
The procedure, which has previously been published by this author [13] for in-plane mountings and is now extended to off-plane ones also, is best explained by a numerical example:

Consider an Ebert-Fastie spectrograph of $r=2 \mathrm{~m}$ mirror radius, $H=80 \mathrm{~mm}$ grating height, and $L=360$ $\mathrm{mm}$ spectrum length. Thus, with $(37 \mathrm{a}, \mathrm{b})$,

$$
\gamma=0.05, \alpha_{\max }= \pm 0.2,
$$

and, with (39a) and (40),

$$
\begin{array}{r}
x=\left[(999.167+2.884 \epsilon / r)-(0.129-1731.381 \epsilon / r) \alpha^{2}\right. \\
\left.\left.-(115.100+309.401 \epsilon / r) \alpha^{4}\right] \mathrm{~mm}, \quad(41)\right)
\end{array}
$$

where linear terms in $\epsilon / r$ are retained, only. Hence, for the spectrum center $(\alpha=\gamma=0.05)$,

$$
x_{0}=(999.166+1.442 \epsilon / r) \mathrm{mm},
$$

and, for the spectrum ends $\left(\alpha=\alpha_{\max }= \pm 0.2\right)$,

so that

$$
x_{\max }=(998.978+65.862 \epsilon / r) \mathrm{mm},
$$

$$
\epsilon / r=0.002918, \epsilon=5.836 \mathrm{~mm},
$$

if one demands that $x_{0}$ and $x_{\max }$ be equal.

The focal curves obtained from (39 a and b) for the uncorrected and the corrected $\sqrt{3}$-position of the grating $(m=1154.700 \mathrm{~mm}$, and $1160.536 \mathrm{~mm}$, respectively) are shown in figure 7 . For the latter,

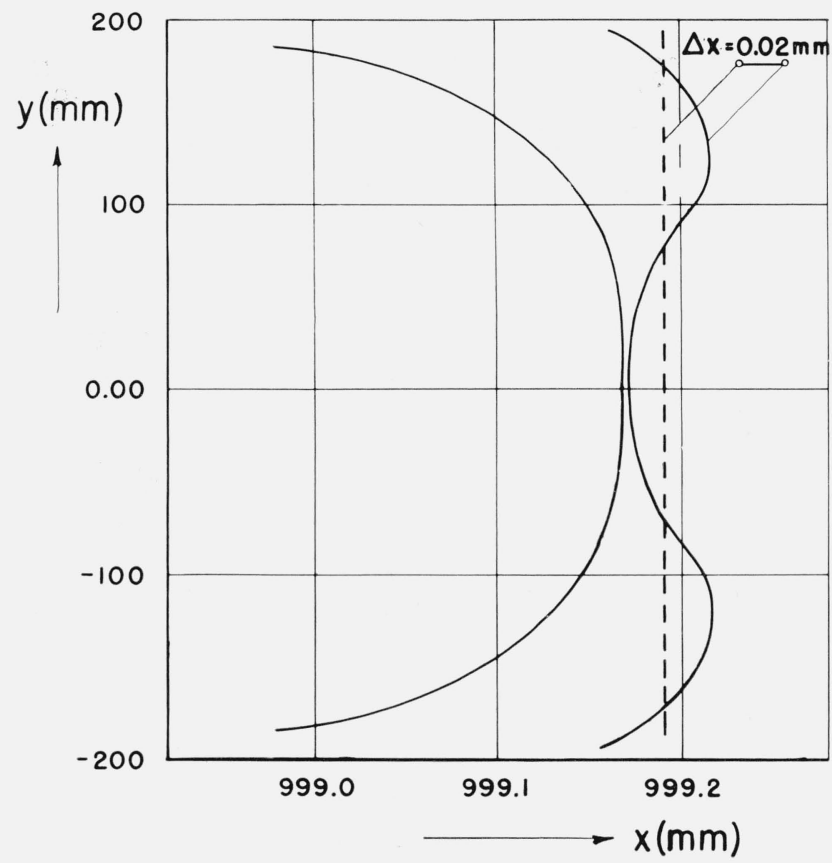

Figure 7. Horizontal cross sections of focal surfaces of EbertFastie spectograph.

Left: uncorrected, right: corrected $\sqrt{ } 3$-position of grating. Scale of abscissa the position of the photographic plate may be chosen as indicated by the broken line. In our example, a displacement of the grating of about $6 \mathrm{~mm}$ has thus resulted in a residual curvature of the spectrum of only $\triangle x=0.02 \mathrm{~mm}$.

For the uncorrected $\sqrt{3}$-position, the residual curvature is of the order of $0.1 \mathrm{~mm}$ and so is on the verge of being acceptable without further improvement [13]. Also, the above example involves a rather extreme relative spectrum length, where image quality at the ends of the spectrum, rather than deviation from flatness, is likely to be the chief consideration.

It may therefore again be concluded that the fourth-order approximation of the focal surface will be needed only rarely.

\section{Conclusions}

The chief results of the above theory of Ebert and Ebert-Fastie systems, and their consequences, may be summarized as follows.

(1) Closed expressions for the focal surfaces and slit curves can be derived in a second-order approximation only, but the accuracy obtained with it is sufficient in most practical cases.

(2) The curvature of the focal surface is strongly dependent upon the position of the grating in the spectrograph. An extended flat spectrum is obtained when the grating is arranged at the $\sqrt{3}$-position $(28)$, which may be slightly corrected for still better flatness.

(3) The off-plane Ebert-Fastie system is the preferred mounting for spectrographs as it yields, other parameters equal, a spectrum twice as long as the in-plane Ebert system.

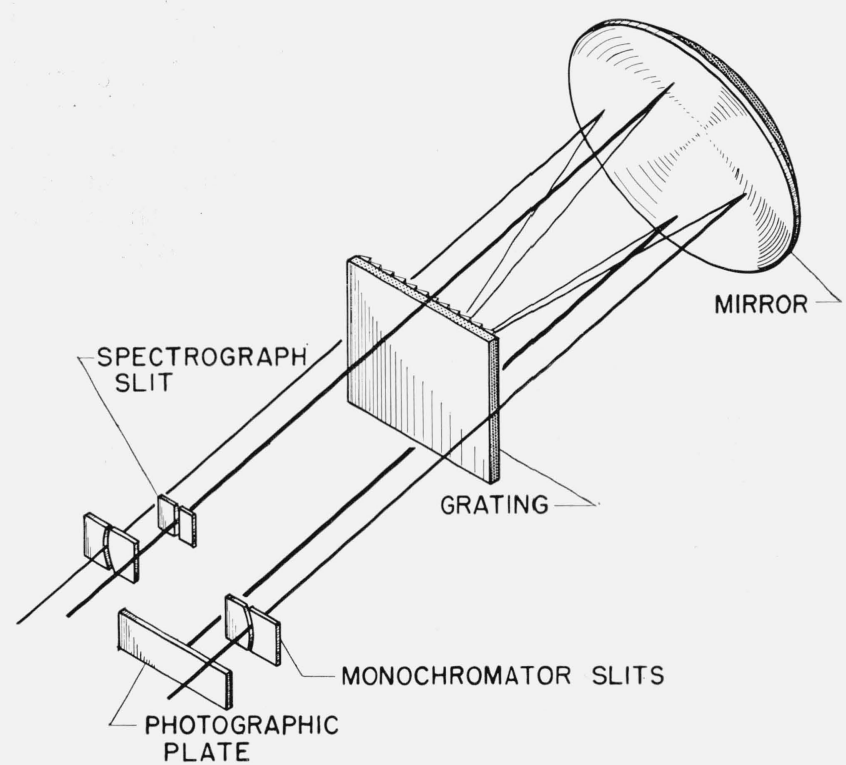

FIGURE 8. Ebert-Fastie spectrograph combined with Ebert monochromator. 
(4) Long slits, as required to maximize the energy throughput of monochromators, must be curved but can be used in an in-plane arrangement only. The Ebert system is therefore the preferred mounting for monochromators.

(5) These relative merits of the two mountings immediately suggest the combined spectrographmonochromator system of figure 8 , where the mere addition of two curved monochromator slits to an Ebert-Fastie spectrograph converts the instrument into an Ebert monochromator as well. A versatile double-purpose system is obtained in this manner at little extra cost and effort. G. W. King [14] has actually built such an instrument.

The programming of numerical computations needed for this paper was done by J. J. Spijkerman, of NBS.

(Paper 68C4-165)

\section{References}

[1] K. D. Mielenz, Theory of mirror spectrographs I. Astigmatic illumination of plane gratings and prisms, J. Res. NBS 68C (Eng. and Instr.), No. 4, 195 (Oct.-Dec. 1964).

[2] K. D. Mielenz, Theory of mirror spectrographs II. General theory of focal surfaces and slit curvatures, 68C (Eng. and Instr.), No. 4, 201 (Oct.-Dec. 1964).

[3] H. Ebert, Wiedem. Ann. 38, 489 (1889).

[4] R. F. Jarrell, J. Opt. Soc. Am. 45, 259 (1955).

[5] K. Rottmann, Math. Formelsamml., p. 60 ff. (Bibliogr. Inst., Mannheim, 1961).

[6] I. S. Sokolnikoff and R. M. Redheffer, Math. of Physics and Mod. Engineering, p. 309 (McGraw-Hill Book Co., New York, N.Y., 1958).

[7] O. Vierle, Dissertation TH Munich, 1962.

[8] S. A. Khrshanovskii, Opt. Spectr. 9, 207 (1960).

[9] W. G. Fastie, J. Opt. Soc. Am. 42, 641 (1952).

[10] W. G. Fastie, J. Opt. Soc. Am. 42, 647 (1952).

[11] P. Kroeplin, Optik aller Wellenlaengen, p. 123 (Akademie-Verlag, Berlin 1959).

[12] H. Kaiser, K. D. Mielenz, and F. Rosendahl, Z. Instr. kde. 6\%, 269 (1959).

[13] K. D. Mielenz, Optik 20, 28 (1963).

[14] G. W. King, J. Sci. Instr. 35, 11 (1958). 